\title{
SLIP FLOW OF A SPHERE IN NON-CONCENTRIC SPHERICAL HYPOTHETICAL CELL
}

\author{
Krishna Prasad Madasu \\ Department of Mathematics, National Institute of Technology, Raipur-492010 \\ Chhatisgarh, India \\ madaspra.maths@nitrr.ac.in,kpm973@gmail.com
}

Received: 23 May 2020; Accepted: 19 August 2020

\begin{abstract}
Slow axisymmetric flow of an incompressible viscous fluid caused by a slip sphere within a non-concentric spherical cell surface is investigated. The uniform velocity (Cunningham's model) and tangential velocity reaches minimum along a radial direction are imposed conditions at the cell surface (Kvashnin's model). The general solution of the problem is combined using superposition of the fundamental solution in the two spherical coordinate systems based on the centers of the slip sphere and spherical cell surface. Numerical results for the correction factor on the inner sphere are obtained with good convergence for various values of the relative distance between the centers of the sphere and spherical cell, the slip coefficient, and the volume fraction. The obtained results are in good agreement with the published results. The effect of concentration is more in the Cunningham's model compared to the Kvashnin's model. The wall correction factor on the no-slip sphere is more compared to that of a slip sphere. The correction factor on the slip sphere is more than that of a spherical gas bubble.
\end{abstract}

MSC 2010: 76A05, 76D07, 76S05

Keywords: Stokes flow, rigid sphere, non-concentric, drag force, slip condition

\section{Introduction}

Several researchers have investigated the fluid flow past a solid particle with slip condition at a fluid-solid interface due to the assumption of a no-slip boundary condition at the fluid-solid interface which is not valid in the case of micro and nano-fluidic devices [1]. In real life applications, the solid particles are not isolated. They form an assemblage of particles. The presence of a neighboring wall or a particle will affect the movement of an inner particle significantly. It is very difficult to study this type of problem due to complex geometry. The essence of the problem is to assume a single particle in a cell and solve it. Ramkissoon and Rahaman [2] investigated the slow motion of a spheroidal particle in a concentric spherical container using slip at the surface of the particle. The different features of the hydrodynamic cell models are discussed by Zholkovskiy et al. [3]. Sherief et al. [4] solved the problem of 
steady translational motion of spherical or spheroidal particle in a cell model using linear slip and microrotation slip conditions. Srinivasacharya and Krishna Prasad [5] examined the Stokes flow past a porous approximate sphere fixed with a solid core surrounded by a spherical envelope. Saad [6] discussed an application of Stokes flow through a porous prolate and an oblate spheorid in cell models.

Gluckman et al. [7] proposed a boundary collocation method for slow viscous flow past a finite assemblage of particles. Using this multipole truncation technique, many researchers solved multi-particle interaction, particle-wall boundaries, and non-concentrated systems (Leichtberg et al. [8], Ganatos et al. [9, 10], Keh and Lee [11]). Faltas and Saad [12] investigated the slow motion of a slip sphere in an eccentric cell using Happel and Kuwabara boundary conditions. Saad [13] discussed the analytical solutions for the problems of slow flow of a micropolar fluid sphere past a viscous fluid sphere and vice-versa. A series of research work is done by assuming the inner particle as a solid or a porous filled with Newtonian fluid so that the center of the particle is located away from the center of the spherical cell [14-17]. Recently, Sherief et al. [18] investigated the translational and rotational motion of a no slip sphere within a non-concentric spherical cavity. Krishna Prasad [19] handled the problem of cell models for non-Newtonian fluid past a semipermeable sphere. Tseng and Keh [20] investigated the quasi-steady thermophoresis of an aerosol sphere located arbitrarily in a spherical cavity normal to the line of their centers. Alouges et al. [21] studied the motion of a solid particle in a bounded viscous flow using the Sparse Cardinal Sine Decomposition.

The purpose of present paper is to fill the gap of literature with previous investigation of Faltas and Saad [12] by presenting the analytical and numerical study of slow flow caused by a solid sphere with a slip flow surface moving in a viscous fluid in an eccentric spherical cell using Cunningham's (Mehta and Morse's) [22, 23] and Kvashnin's [24] boundary conditions. The wall correction factor is calculated numerically and it agrees with the analytical results of the concentric case. The effects of dimensionless parameters are discussed in detail.

\section{Mathematical statement}

As depicted in Figure 1, consider the slow motion of a sphere with radius $a$ in an incompressible viscous fluid, surrounded by a non-concentric spherical cell with radius $b$. The spherical cell is frictionless. The fluid comes near the cell surface and past a sphere translating at a constant velocity $U$ from the negative $z$-axis. The particle volume fraction of the sphere is equal to the particle volume fraction throughout the cavity ( $\gamma=\eta^{3}$ where $\eta=\frac{a}{b}$ is the separation parameter). Here $(r, \theta, \phi)$ and $(\rho, \phi, z)$, denote the spherical coordinate and circular cylindrical systems, respectively.

The origin is fixed at the centre of spherical cell. The centre of the sphere is placed at a distance $d$ from the centre of the cell. Let $\left(r_{1}, \theta_{1}, \phi_{1}\right)$ and $\left(r_{2}, \theta_{2}, \phi_{2}\right)$ be the spher- 
ical coordinates based on the centre of a inner sphere and outer spherical cavity, respectively. The relation between the radii of a sphere $r_{1}$ and spherical cell $r_{2}$ are given by $r_{1}^{2}=r_{2}^{2}+d^{2}-2 r_{2} d \cos \theta_{2}$ or $r_{2}^{2}=r_{1}^{2}+d^{2}+2 r_{1} d \cos \theta_{1}$.

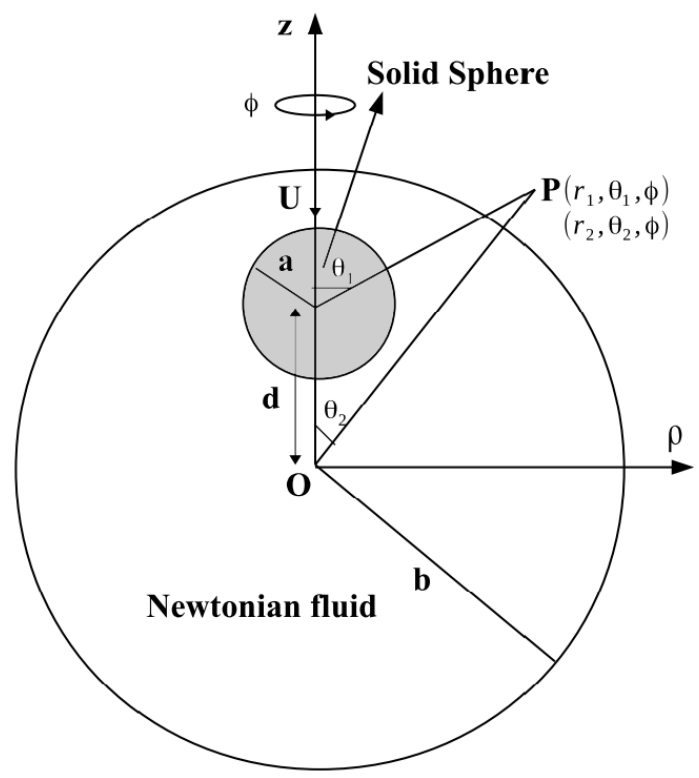

Fig. 1. The physical situation of slip sphere in a non-concentric spherical hypothetical cell

The equations governing the motion are given by

$$
\begin{gathered}
\nabla \cdot \overrightarrow{\mathbf{V}}=0 \\
\nabla p+\mu \nabla \times \nabla \times \overrightarrow{\mathbf{V}}=0 .
\end{gathered}
$$

where $\overrightarrow{\mathbf{V}}, p$ are the velocity vector and fluid pressure at any point, and $\mu$ is the viscosity coefficient.

The flow generated is axially symmetric and all the quantities are independent of $\phi$. Thus, one can take the velocity vector in the cylindrical coordinates as

$$
\overrightarrow{\mathbf{V}}=\mathbf{V}_{\rho}(\rho, z) \vec{e}_{\rho}+\mathbf{V}_{z}(\rho, z) \vec{e}_{z}
$$

Since $\nabla \cdot \overrightarrow{\mathbf{V}}=0$, one can represent the velocity components $q_{\rho}$ and $q_{z}$ in terms of the Stokes stream function as

$$
\mathbf{V}_{\rho}=\frac{1}{\rho} \frac{\partial \psi}{\partial z}, \quad \mathbf{V}_{z}=-\frac{1}{\rho} \frac{\partial \psi}{\partial \rho}
$$


After elimination of pressure from (2), we get

$$
E^{4} \Psi=0
$$

Where

$$
E^{2}=\frac{\partial^{2}}{\partial \rho^{2}}-\frac{1}{\rho} \frac{\partial}{\partial \rho}+\frac{\partial^{2}}{\partial z^{2}} \quad \text { is the Stokesian operator. }
$$

For flow configuration, the boundary conditions along the surface of slip sphere $r_{1}=a[11,12,25]$.

$$
\begin{gathered}
\lambda \mathbf{V}_{\rho}=t_{r_{1} \theta_{1}} \xi_{1} \\
\lambda \mathbf{V}_{z}=-t_{r_{1} \theta_{1}}\left(1-\xi_{1}^{2}\right)^{1 / 2}
\end{gathered}
$$

Where $\xi_{1}=\cos \theta_{1}, t_{r_{1} \theta_{1}}$ is the stress tensor for the flow, and $\lambda$ is the slip coefficient. The slip sphere behaves as spherical gas bubble if $\lambda=0$ and slip sphere becomes no slip sphere if $\lambda \rightarrow \infty$.

On the spherical hypothetical cell surface $r_{2}=b$, the continuity of radial velocity along with homogeneity of the flow and symmetry of the cell.

$$
\mathbf{V}_{\rho}\left(1-\xi_{2}^{2}\right)^{1 / 2} \xi_{2}^{-1}+\mathbf{V}_{z}=-1
$$

\section{Cunningham's (Mehta-Morse) model:}

$$
\mathbf{V}_{\rho} \xi_{2}\left(1-\xi_{2}^{2}\right)^{-1 / 2}-\mathbf{V}_{z}=1
$$

\section{Kvashnin's model:}

$$
\frac{\partial}{\partial r}\left(\mathbf{V}_{\rho}-\mathbf{V}_{z}\left(1-\xi_{2}^{2}\right)^{1 / 2} \xi_{2}^{-1}\right)=0
$$

\section{Solution of the problem}

Using the principle of superposition, the stream function $\Psi$ of the fluid flow in the spherical coordinates is given by $[11,12,25]$

$$
\Psi=\sum_{n=2}^{\infty}\left[\left(\mathbf{A}_{n} r_{1}^{-n+1}+\mathbf{B}_{n} r_{1}^{-n+3}\right) \vartheta_{n}\left(\xi_{1}\right)+\left(\mathbf{C}_{n} r_{2}^{n}+\mathbf{D}_{n} r_{2}^{n+2}\right) \vartheta_{n}\left(\xi_{2}\right)\right]
$$

where $\xi_{1}=\cos \theta_{1}, \xi_{2}=\cos \theta_{2}, \vartheta_{n}(\cdot)$ is the Gegenbauer function of the first kind of order $n$ and degree $-1 / 2$. The unknown constants $\mathbf{A}_{n}, \mathbf{B}_{n}, \mathbf{C}_{n}$, and $\mathbf{D}_{n}$ are to be determined using boundary conditions Eqs. (6)-(10).

The expressions for the axial velocity $\mathbf{V}_{\rho}$, the radial velocity $\mathbf{V}_{z}$, and the stress tensor $t_{r_{1}} \theta_{1}$ are given by 


$$
\begin{aligned}
& \mathbf{V}_{\rho}=\sum_{n=2}^{\infty}\left[\mathbf{A}_{n} A_{1 n}\left(\zeta_{1}\right)+\mathbf{B}_{n} B_{1 n}\left(\zeta_{1}\right)+\mathbf{C}_{n} C_{1 n}\left(\zeta_{2}\right)+\mathbf{D}_{n} D_{1 n}\left(\zeta_{2}\right)\right] \\
& \mathbf{V}_{z}=\sum_{n=2}^{\infty}\left[\mathbf{A}_{n} A_{2 n}\left(\zeta_{1}\right)+\mathbf{B}_{n} B_{2 n}\left(\zeta_{1}\right)+\mathbf{C}_{n} C_{2 n}\left(\zeta_{2}\right)+\mathbf{D}_{n} D_{2 n}\left(\zeta_{2}\right)\right] \\
& t_{r_{1} \theta_{1}}=\sum_{n=2}^{\infty}\left[\mathbf{A}_{n} A_{3 n}\left(\zeta_{1}\right)+\mathbf{B}_{n} B_{3 n}\left(\zeta_{1}\right)+\mathbf{C}_{n} C_{3 n}\left(\zeta_{2}\right)+\mathbf{D}_{n} D_{3 n}\left(\zeta_{2}\right)\right]
\end{aligned}
$$

Where the points $\zeta_{1}=\left(r_{1}, \theta_{1}\right), \zeta_{2}=\left(r_{2}, \theta_{2}\right)$, the functions $A_{k n}, B_{k n}, C_{k n}$ and $D_{k n}$ with $k=1,2,3$ are given in the Appendix.

For Cunningham's model, the boundary conditions (6)-(9) are applied

$$
\begin{aligned}
& \sum_{n=2}^{\infty}\left[\mathbf{A}_{n} A_{4 n}\left(\zeta_{3}\right)+\mathbf{B}_{n} B_{4 n}\left(\zeta_{3}\right)+\mathbf{C}_{n} C_{4 n}\left(\zeta_{4}\right)+\mathbf{D}_{n} D_{4 n}\left(\zeta_{4}\right)\right]=0 \\
& \sum_{n=2}^{\infty}\left[\mathbf{A}_{n} A_{5 n}\left(\zeta_{3}\right)+\mathbf{B}_{n} B_{5 n}\left(\zeta_{3}\right)+\mathbf{C}_{n} C_{5 n}\left(\zeta_{4}\right)+\mathbf{D}_{n} D_{5 n}\left(\zeta_{4}\right)\right]=0 \\
& \sum_{n=2}^{\infty}\left[\mathbf{A}_{n} A_{6 n}\left(\zeta_{5}\right)+\mathbf{B}_{n} B_{6 n}\left(\zeta_{5}\right)+\mathbf{C}_{n} C_{6 n}\left(\zeta_{6}\right)+\mathbf{D}_{n} D_{6 n}\left(\zeta_{6}\right)\right]=-1 \\
& \sum_{n=2}^{\infty}\left[\mathbf{A}_{n} A_{7 n}\left(\zeta_{5}\right)+\mathbf{B}_{n} B_{7 n}\left(\zeta_{5}\right)+\mathbf{C}_{n} C_{7 n}\left(\zeta_{6}\right)+\mathbf{D}_{n} D_{7 n}\left(\zeta_{6}\right)\right]=1
\end{aligned}
$$

where the points $\zeta_{3}=\left(1, \theta_{1}\right), \zeta_{4}=\left[\left(r_{2}, \theta_{2}\right)\right]_{r_{1}=1}, \zeta_{5}=\left[\left(r_{1}, \theta_{1}\right)\right]_{r_{2}=\frac{1}{\eta}}, \zeta_{6}=\left(\frac{1}{\eta}, \theta_{2}\right)$ and for Kvashnin model, Eq. (9) is replaced by Eq. (10)

$$
\sum_{n=2}^{\infty}\left[\mathbf{A}_{n} A_{8 n}\left(\zeta_{5}\right)+\mathbf{B}_{n} B_{8 n}\left(\zeta_{5}\right)+\mathbf{C}_{n} C_{8 n}\left(\zeta_{6}\right)+\mathbf{D}_{n} D_{8 n}\left(\zeta_{6}\right)\right]=0
$$

where the functions $\mathbf{A}_{k n}, \mathbf{B}_{k n}, \mathbf{C}_{k n}$, and $\mathbf{D}_{k n}$ with $k=4,5,6,7,8$ are given in the Appendix.

To obtain the fully converged velocity components, the boundary conditions (6)-(9) or (6)-(8) and (10) are to be fulfilled along the surface of slip sphere and outer cell boundary. These equations constitute a set of linear equations with an infinite number of unknown coefficients, and it cannot be solved. To overcome this difficulty, the multipole collocation method is used. The infinite series should be truncated after a finite number of terms $N$ so that the number of the unknown coefficients becomes finite. A finite number $N$ of collocation points are to be chosen at both the slip sphere 
and spherical hypothetical cell so that one can have same number of linear equations and unknown coefficients. To achieve the desired accuracy, a large number of collocation points are used.

\section{Drag on the slip sphere}

The drag force can be calculated by using the formula

$$
F=\left.\pi \mu a \int_{0}^{\pi} r^{3} \sin ^{3} \theta \frac{\partial}{\partial r}\left(\frac{E^{2} \Psi}{r^{2} \sin ^{2} \theta}\right) r\right|_{r=1} d \theta=-4 \pi \mu a U B_{2}
$$

The Eq. (20) indicates that $B_{2}$ contributes to the hydrodynamic force experienced by the slip sphere. The expression $B_{2}$ is the lowest order coefficient. The value of $B_{2}$ is the most precise and quickest convergent result.

If the cell surface is absent i.e., $\frac{a}{(b-d)}=0$, the fluid is unbounded. The drag acting on a slip sphere in a viscous fluid is given as

$$
\mathscr{F}_{\infty}=-6 \pi a U \mu\left[\frac{\lambda_{1}+2}{\lambda_{1}+3}\right]
$$

Where $\lambda_{1}=\lambda a / \mu$.

Using Eqs. (20) and (21), the wall effect $W$ for the slow flow of slip sphere is given by

$$
W=\frac{\mathscr{F}}{\mathscr{F}_{\infty}} .
$$

In the absence of a hypothetical cell surface, $W=1$.

The solution for the slow motion of a slip sphere located at the center of a spherical hypothetical cell for both models are

\section{Cunningham's model:}

$$
W_{C u}=-\left[\frac{\lambda_{1}+3}{\lambda_{1}+2}\right] \frac{\left[\left(\lambda_{1}-3\right) \gamma^{5 / 3}-\left(\lambda_{1}+2\right)\right]}{\left[\left(\lambda_{1}-3\right) \gamma^{2}-\frac{9}{4}\left(\lambda_{1}-2\right) \gamma^{5 / 3}+\frac{5}{2} \gamma \lambda_{1}-\frac{9}{4}\left(\lambda_{1}+2\right) \gamma^{1 / 3}+\left(\lambda_{1}+3\right)\right]}
$$

\section{Kvashnin's model:}

$W_{K v}=-\left[\frac{\lambda_{1}+3}{\lambda_{1}+2}\right] \frac{\left[\left(\lambda_{1}-3\right) \gamma^{5 / 3}+4\left(\lambda_{1}+2\right)\right]}{\left[2\left(\lambda_{1}-3\right) \gamma^{2}-\frac{9}{4}\left(\lambda_{1}-2\right) \gamma^{5 / 3}-\frac{5}{2} \gamma \lambda_{1}+\frac{27}{4}\left(\lambda_{1}+2\right) \gamma^{1 / 3}-4\left(\lambda_{1}+3\right)\right]}$ 


\begin{tabular}{|c|c|}
\hline no slip $\lambda_{1} \rightarrow \infty$ & perfect slip $\lambda_{1} \rightarrow 0$ \\
\hline$W_{C u}=-\frac{1-\gamma^{5 / 3}}{\gamma^{2}-\frac{9}{4} \gamma^{5 / 3}+\frac{5}{2} \gamma-\frac{9}{4} \gamma^{1 / 3}+1}$ & $W_{C u}=-\frac{1+\frac{3}{2} \gamma^{5 / 3}}{\gamma^{2}-\frac{3}{2} \gamma^{5 / 3}+\frac{3}{2} \gamma^{1 / 3}-1}$ \\
\hline$W_{K v}=-\frac{4+\gamma^{5 / 3}}{2 \gamma^{2}-\frac{9}{4} \gamma^{5 / 3}-\frac{5}{2} \gamma+\frac{27}{4} \gamma^{1 / 3}-4}$ & $W_{K v}=-\frac{1+\frac{3}{8} \gamma^{5 / 3}}{\frac{1}{2} \gamma^{2}-\frac{3}{8} \gamma^{5 / 3}-\frac{9}{8} \gamma^{1 / 3}+1}$ \\
\hline
\end{tabular}

\section{Numerical results}

To obtain the numerical solution of the wall correction factor $W$ for a slip sphere translating within a spherical cell, choose the boundary collocation points along the half-circular generating arcs of the slip sphere and spherical cell. The initial point $\theta_{i}=0$ and the terminal point $\theta_{i}=\pi$ are chosen along with the point $\theta_{i}=\pi / 2$ on both the arcs. If these three points are used in the system (15)-(17), Eq. (18) and (19) for Cunningham's and Kvashnin's models, respectively, the coefficient matrix becomes a singular matrix. To avoid this difficulty and achieve good accuracy, the method recommended in literature [7,9-12] is used. The adjacent points of $\theta_{i}=0$, $\pi / 2, \pi$ are $\theta_{i}=\varepsilon, \theta_{i}=\pi / 2-\varepsilon, \theta_{i}=\pi / 2+\varepsilon, \theta_{i}=\pi-\varepsilon$ where $\varepsilon$ is a specified value $\left(\varepsilon=0.01^{\circ}\right.$ ) and is chosen so that the coefficient matrix is nonsingular. Divide the two quarter-circular arcs into equal segments so that additional points are selected as mirror-image pairs about $\theta_{i}=\pi / 2$. Choosing a finite number of discrete points on the arcs results in a system of linear equations. This system is solved by using the Gaussian elimination method.

Table 1. Convergence of the Wall correction factor $W$ for different values of $\delta, \gamma$, and $\lambda$

\begin{tabular}{llrrrr}
\hline & & \multicolumn{4}{c}{$W$} \\
\cline { 3 - 6 }$\delta$ & $N$ & $\lambda_{1}=1$ & $\lambda_{1}=10$ & $\lambda_{1}=1$ & $\lambda_{1}=10$ \\
& & $\gamma=0.5$ & $\gamma=0.9$ & $\gamma=0.5$ & $\gamma=0.9$ \\
\hline 0.6 & 6 & 13.518643 & 1698.395542 & 141.649642 & 36754.084995 \\
& 10 & 11.964950 & 1306.732609 & 93.319030 & 16392.267391 \\
& 14 & 11.965407 & 1307.992201 & 93.036636 & 16539.270357 \\
& 18 & 11.965422 & 1307.996379 & 93.038305 & 16540.751912 \\
& 22 & 11.965422 & 1307.996398 & 93.038314 & 16540.761573 \\
& 26 & - & 1307.996398 & 93.038314 & 16540.761659 \\
& 30 & - & - & - & 16540.761662 \\
& 34 & - & - & - & 16540.761662 \\
& 36 & - & - & - & - \\
\hline
\end{tabular}

The impact of the slip parameter $\lambda_{1}=\lambda a / \mu$, normalized deviation distance of the center of slip sphere from the center of spherical cell $\delta=\frac{d}{b-a}$, volume fraction $\gamma=\eta^{3}$ on the wall correction factor $W$ are presented in Figure $2 \mathrm{a}$ and $2 \mathrm{~b}$, and Tables 2 and 3. The convergence of the numerical results for the wall correction factor $W$ is tested and tabulated in Table 1 . All the numerical values are accurate to at 
least six decimal places. Figure 2 a demonstrates that $W$ is increasing function of $\gamma$

Table 2. Cunningham's model: Wall correction factor $W_{C u}$ for different values of $\delta$, $\gamma$, and $\lambda$

\begin{tabular}{llrrrr}
\hline & \multicolumn{5}{c}{$W_{C u}$} \\
\cline { 3 - 6 }$\delta$ & $\gamma$ & $\lambda_{1} \rightarrow 0$ & $\lambda_{1}=1$ & $\lambda_{1}=10$ & $\lambda_{1} \rightarrow \infty$ \\
\hline 0.00001 & 0.001 & 1.176469 & 1.202104 & 1.259094 & 1.286193 \\
& 0.1 & 3.16586 & 3.542381 & 4.844169 & 5.852977 \\
& 0.5 & 46.1306 & 47.812792 & 70.535256 & 125.257459 \\
& 0.9 & 11576.44699 & 10564.3528 & 10426.576597 & 31322.214682 \\
0.25 & 0.001 & 1.184106 & 1.212312 & 1.275391 & 1.305576 \\
& 0.1 & 3.219083 & 3.620338 & 5.045277 & 6.20142 \\
& 0.5 & 48.87828 & 50.475502 & 74.183503 & 135.018724 \\
& 0.9 & 12376.295164 & 11283.70343 & 11071.959217 & 33835.488861 \\
0.5 & 0.001 & 1.224763 & 1.260879 & 1.343113 & 1.383221 \\
& 0.1 & 3.422713 & 3.913382 & 5.814358 & 7.609405 \\
& 0.5 & 59.830182 & 61.004396 & 88.174728 & 174.802337 \\
& 0.9 & 15564.382983 & 14147.743805 & 13618.928582 & 44087.677691 \\
\hline
\end{tabular}

and becomes infinite as $\gamma$ approaches to 1 for fixed values of $\delta$ and $\lambda_{1}$. The wall correction factor exerted on the slip sphere by the fluid increases with an increase in the slip parameter $\lambda_{1}$. The effect of concentration is more in the Cunningham's model compared to the Kvashnin's model. For specified values of $\gamma$ and $\delta$, the wall effect on the no-slip sphere is more compared to that of a slip sphere. The correction factor on the slip sphere is more than that of a spherical gas bubble. Figure $2 \mathrm{~b}$ illustrates

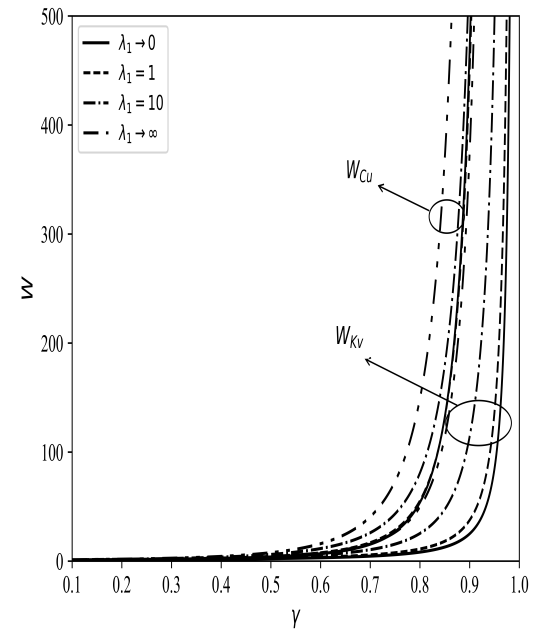

(a) For different values of $\lambda$ with $\delta=0.25$

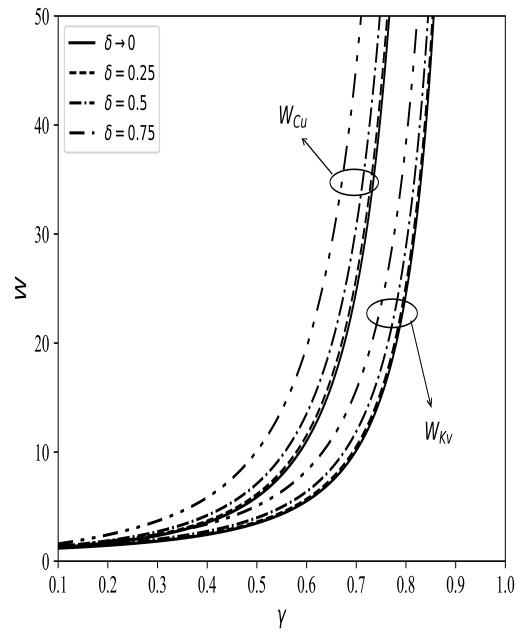

(b) For different values of the parameter $\delta$ with slip parameter $\lambda=10$

Fig. 2. Variation of $W$ versus $\gamma$ 
that $W$ increases monotonically with an increase in $\delta$, for any fixed values of $\gamma$ and $\lambda_{1}$. The numerical values of the $W$ are presented in Tables 1 and 2 for Cunningham's model and Kvashnin's model, respectively. If $\delta \rightarrow 0$, the center of a slip sphere and spherical hypothetical cell coincide, and the problem is reduced to translation of a slip sphere in a concentric spherical container.

In Tables 2 and 3, the numerical values of $W$ for $\delta \rightarrow 0$ agree with analytical solutions (23) and (24). Comparing the present tabular values with given data in the work by Faltas and Saad [12], the wall correction factor decreases in the following direction from Cunningham's model, to Kuwabara's model [12], Kvashnin's model and Happel's model [12]. The wall correction factor is higher for Cunningham's model when compared with other cell models. The behaviour of flow parameters is the same in all four models.

Table 3. Kvashnin's model: Wall correction factor $W_{K \nu}$ for different values of $\delta, \gamma$, and $\lambda$

\begin{tabular}{llrrrr}
\hline & \multicolumn{5}{c}{$W_{K v}$} \\
\cline { 3 - 6 }$\delta$ & $\gamma$ & $\lambda_{1} \rightarrow 0$ & $\lambda_{1}=1$ & $\lambda_{1}=10$ & $\lambda_{1} \rightarrow \infty$ \\
\hline 0.00001 & 0.001 & 1.126761 & 1.144696 & 1.183833 & 1.202097 \\
& 0.1 & 2.089392 & 2.329996 & 3.041432 & 3.510962 \\
& 0.5 & 7.737922 & 10.320974 & 22.853293 & 42.62212 \\
& 0.9 & 162.526198 & 268.824419 & 1032.777179 & 8189.195994 \\
0.25 & 0.001 & 1.131892 & 1.151292 & 1.193783 & 1.213688 \\
& 0.1 & 2.103138 & 2.35818 & 3.126784 & 3.649964 \\
& 0.5 & 7.868034 & 10.549205 & 23.728373 & 45.53468 \\
& 0.9 & 167.36303 & 277.372406 & 1070.083372 & 8864.609211 \\
0.5 & 0.001 & 1.156683 & 1.180622 & 1.233649 & 1.258784 \\
& 0.1 & 2.14934 & 2.456677 & 3.443746 & 4.191274 \\
& 0.5 & 8.327333 & 11.363381 & 26.933211 & 57.316415 \\
& 0.9 & 184.82683 & 308.298498 & 1205.58458 & 11642.401731 \\
\hline
\end{tabular}

\section{Conclusion}

In this work, the slow motion of a slip sphere in a spherical hypothetical cell is studied analytically and numerically by using the multipole collocation technique. The wall effect of a slip sphere is calculated for different values of the slip parameter, volume fraction of the dispersed system, and the normalized deviation distance of the center of slip sphere from the center of spherical hypothetical cell. The results show that the solution procedure converges rapidly for a greater number of collocation points. It is observed that the wall factor is an increasing function of the slip parameter, normalized deviation distance between centers of the slip sphere and the cell surface, and volume fraction. The boundary effect is minimal when the particle located at center of hypothetical cell and increases as the distance between the center of slip sphere located away from the center of cell surface. It is found that the wall 
effect on the no-slip sphere is more compared to that of a slip sphere and the effect on the slip sphere is more compared to that of a spherical gas bubble.

\section{References}

[1] Neto, C., Evans, D.R., Bonaccurso, E., Butt, H.J., \& Craig, V.S.J. (2005). Boundary slip in Newtonian liquids: a review of experimental studies. Reports on Progress in Physics, 68, 2859-2897.

[2] Ramkissoon, H., \& Rahaman, K. (2003). Wall effects with slip. Journal of Applied Mathematics and Mechanics, 83(11), 773-778.

[3] Zholkovskiy, E.K., Shilov, V.N., Masliyah, J.H., \& Bondarenko, M.P. (2007). Hydrodynamic cell model: General formulation and comparative analysis of different approaches. The Canadian Journal of Chemical Engineering, 85, 701-725.

[4] Sherief, H.H., Faltas, M.S., Ashmawy, E.A., \& Nashwan, M.G. (2015). Stokes flow of a micropolar fluid past an assemblage of spheroidal particle-in-cell models with slip. Physica Scripta, 90(5), 055203.

[5] Srinivasacharya, D., \& Krishna Prasad, M. (2012). Creeping motion of a porous approximate sphere with an impermeable core in a spherical container. European Journal of Mechanics B/Fluids, 36, 104-114.

[6] Saad, E. (2012). Stokes flow past an assemblage of axisymmetric porous spheroidal particle-in-cell models. Journal of Porous Media, 15(9), 849-866.

[7] Gluckman, M.J., Pfeffer, R., \& Weinbaum, S. (1971). A new technique for treating multiparticle slow viscous flow: axisymmetric flow past spheres and spheroids. Journal of Fluid Mechanics, $50,705-740$.

[8] Leichtberg, S., Pfeffer, R., \& Weinbaum, S. (1976). Stokes flow past finite coaxial clusters of spheres in a circular cylinder. International Journal of Multiphase Flow, 3, 147-169.

[9] Ganatos, P., Weinbaum, S., \& Pfeffer, R. (1980). A strong interaction theory for the creeping motion of a sphere between plane parallel boundaries. Part 1. Perpendicular motion. Journal of Fluid Mechanics, 99, 739-753.

[10] Ganatos, P., Weinbaum, S., \& Pfeffer, R. (1980). A strong interaction theory for the creeping motion of a sphere between plane parallel boundaries. Part 2. Parallel motion. Journal of Fluid Mechanics, 99, 755-783.

[11] Keh, H.J., \& Lee, T.C. (2010). Axisymmetric creeping motion of a slip spherical particle in a nonconcentric spherical cavity. Theoretical and Computational Fluid Dynamics, 24, 497-510.

[12] Faltas, M.S., \& Saad, E.I. (2011). Stokes flow past an assemblage of slip eccentric spherical particle-in-cell models. Mathematical Methods in the Applied Sciences, 34, 1594-1605.

[13] Saad, E. (2012). Cell models for micropolar flow past a viscous fluid sphere. Meccanica, 47, 2055-2068.

[14] Faltas, M.S., \& Saad, E.I. (2012). Slow motion of a porous eccentric spherical particle-in-cell models. Transport in Porous Media, 95, 133-150.

[15] Lee, T.C., \& Keh, H.J. (2013). Slow motion of a spherical particle in a spherical cavity with slip surfaces. International Journal of Engineering Science, 69, 1-15.

[16] Saad, E.I. (2014). Motion of a slip sphere in a nonconcentric fictitious spherical envelope of micropolar fluid. ANZIAM Journal, 55, 383-401.

[17] Saad, E.I. (2019). Viscous flow past a porous sphere within a nonconcentric fictitious spherical cell. Microsystem Technologies, 25, 1051-1063. 
[18] Sherief, H.H., Faltas, M.S., El-Sapa, S. (2019). Axisymmetric creeping motion caused by a spherical particle in a micropolar fluid within a nonconcentric spherical cavity. European Journal of Mechanics-B Fluids, 77, 211-220.

[19] Krishna Prasad, M. (2019). Cell models for Non-Newtonian fluid past a semipermeable sphere. International Journal of Mathematical, Engineering and Mangement Sciences, 4(6), 1352-1361.

[20] Tseng, Y.M., \& Keh, H.J. (2020). Thermophoretic motion of an aerosol sphere in a spherical cavity. European Journal of Mechanics/B Fluids, 81, 93-104.

[21] Alouges, F., Lepot, A.L., \& Sellier, A. (2020). Motion of a solid particle in a bounded viscous flow using the Sparse Cardinal Sine Decomposition. Meccanica, 55, 403-419.

[22] Cunningham, E. (1910). On the velocity of steady fall of spherical particles through fluid medium. Proceedings of the Royal Society of London, A 83, 357-365.

[23] Mehta, G.D., \& Morse, T.F. (1975). Flow through charged membranes. Journal of Chemical Physics, 63, 1878-1889.

[24] Kvashnin, A.G. (1979). Cell model of suspension of spherical particles. Fluid Dynamics, 14, 598-602.

[25] Happel, J., \& Brenner, H. (1965). Low Reynolds Number Hydrodynamics. Englewood Cliffs, N.J.: Prentice-Hall.

\section{Appendix A}

$$
\begin{aligned}
& A_{1 n}(\zeta)=-r^{-n-1} Q_{1}, \quad \text { where } \quad Q_{1}=(n+1) \vartheta_{n+1}(\xi)\left(1-\xi^{2}\right)^{-1 / 2} \\
& B_{1 n}(\zeta)=-r^{-n+1}\left(Q_{1}-2 Q_{2}\right), \quad \text { where } \quad Q_{2}=\vartheta_{n}(\xi) \xi\left(1-\xi^{2}\right)^{-1 / 2} \\
& C_{1 n}(\zeta)=-r^{n-2}\left(Q_{1}-(2 n-1) Q_{2}\right) \\
& D_{1 n}(\zeta)=-r^{n}\left(Q_{1}-(2 n+1) Q_{2}\right) \\
& A_{2 n}(\zeta)=-r^{-n-1} P_{n}(\xi) \\
& B_{2 n}(\zeta)=-r^{-n+1}\left[2 \vartheta_{n}(\xi)+P_{n}(\xi)\right] \\
& C_{2 n}(\zeta)=-r^{n-2}\left[(2 n-1) \vartheta_{n}(\xi)+P_{n}(\xi)\right] \\
& D_{2 n}(\zeta)=-r^{n}\left[(2 n+1) \vartheta_{n}(\xi)+P_{n}(\xi)\right] \\
& A_{3 n}(\zeta)=2\left(n^{2}-1\right) r^{-n-2} \vartheta_{n}(\xi)\left(1-\xi^{2}\right)^{-1 / 2} \\
& B_{3 n}(\zeta)=2 n(n-2) r^{-n} \vartheta_{n}(\xi)\left(1-\xi^{2}\right)^{-1 / 2} \\
& C_{3 n}(\zeta)=2 n(n-2) r^{n-3} \vartheta_{n}(\xi)\left(1-\xi^{2}\right)^{-1 / 2} \\
& D_{3 n}(\zeta)=2\left(n^{2}-1\right) r^{n-1} \vartheta_{n}(\xi)\left(1-\xi^{2}\right)^{-1 / 2} \\
& A_{4 n}(\zeta)=A_{1 n}(\zeta)-\lambda_{1}^{-1} \xi_{1} A_{3 n}(\zeta) \\
& B_{4 n}(\zeta)=B_{1 n}(\zeta)-\lambda_{1}^{-1} \xi_{1} B_{3 n}(\zeta) \\
& C_{4 n}(\zeta)=C_{1 n}(\zeta)-\lambda_{1}^{-1} \xi_{1} C_{3 n}(\zeta) \\
& D_{4 n}(\zeta)=D_{1 n}(\zeta)-\lambda_{1}^{-1} \xi_{1} D_{3 n}(\zeta) \\
& A_{5 n}(\zeta)=A_{2 n}(\zeta)+\lambda_{1}^{-1}\left(1-\xi_{1}^{2}\right)^{1 / 2} A_{3 n}(\zeta)
\end{aligned}
$$




$$
\begin{aligned}
B_{5 n}(\zeta) & =B_{2 n}(\zeta)+\lambda_{1}^{-1}\left(1-\xi_{1}^{2}\right)^{1 / 2} B_{3 n}(\zeta), \\
C_{5 n}(\zeta) & =C_{2 n}(\zeta)+\lambda_{1}^{-1}\left(1-\xi_{1}^{2}\right)^{1 / 2} C_{3 n}(\zeta), \\
D_{5 n}(\zeta) & =D_{2 n}(\zeta)+\lambda_{1}^{-1}\left(1-\xi_{1}^{2}\right)^{1 / 2} D_{3 n}(\zeta), \\
A_{6 n}(\zeta) & =A_{1 n}(\zeta)\left(1-\xi_{2}^{2}\right)^{1 / 2} \xi_{2}^{-1}+A_{2 n}(\zeta), \\
B_{6 n}(\zeta) & =B_{1 n}(\zeta)\left(1-\xi_{2}^{2}\right)^{1 / 2} \xi_{2}^{-1}+B_{2 n}(\zeta), \\
C_{6 n}(\zeta) & =C_{1 n}(\zeta)\left(1-\xi_{2}^{2}\right)^{1 / 2} \xi_{2}^{-1}+C_{2 n}(\zeta), \\
D_{6 n}(\zeta) & =D_{1 n}(\zeta)\left(1-\xi_{2}^{2}\right)^{1 / 2} \xi_{2}^{-1}+D_{2 n}(\zeta), \\
A_{7 n}(\zeta) & =A_{1 n}(\zeta) \xi_{2}\left(1-\xi_{2}^{2}\right)^{-1 / 2}-A_{2 n}(\zeta), \\
B_{7 n}(\zeta) & =B_{1 n}(\zeta) \xi_{2}\left(1-\xi_{2}^{2}\right)^{-1 / 2}-B_{2 n}(\zeta), \\
C_{7 n}(\zeta) & =C_{1 n}(\zeta) \xi_{2}\left(1-\xi_{2}^{2}\right)^{-1 / 2}-C_{2 n}(\zeta), \\
D_{7 n}(\zeta) & =D_{1 n}(\zeta) \xi_{2}\left(1-\xi_{2}^{2}\right)^{-1 / 2}-D_{2 n}(\zeta), \\
A_{8 n}(\zeta) & =\frac{\partial}{\partial r}\left(A_{1 n}(\zeta)-A_{2 n}(\zeta)\left(1-\xi_{2}^{2}\right)^{1 / 2} \xi_{2}^{-1}\right), \\
& =(n+1) r^{-n-2}\left(Q_{1}-Q_{3}\right) \quad \text { where } \quad Q_{3}=P_{n}(\xi)\left(1-\xi_{2}^{2}\right)^{1 / 2} \xi_{2}^{-1} \\
& \partial \\
B_{8 n}(\zeta) & \frac{\partial}{\partial r}\left(B_{1 n}(\zeta)-B_{2 n}(\zeta)\left(1-\xi_{2}^{2}\right)^{1 / 2} \xi_{2}^{-1}\right), \\
= & (n-1) r^{-n}\left(Q_{1}-2 Q_{2}-\left(Q_{3}+2 Q_{4}\right)\right)
\end{aligned}
$$$$
C_{8 n}(\zeta)=\frac{\partial}{\partial r}\left(C_{1 n}(\zeta)-C_{2 n}(\zeta)\left(1-\xi_{2}^{2}\right)^{1 / 2} \xi_{2}^{-1}\right)
$$$$
=-(n-2) r^{n-3}\left(Q_{1}-(2 n-1) Q_{2}-Q_{3}-(2 n-1) Q_{4}\right),
$$$$
D_{8 n}(\zeta)=\frac{\partial}{\partial r}\left(D_{1 n}(\zeta)-D_{2 n}(\zeta)\left(1-\xi_{2}^{2}\right)^{1 / 2} \xi_{2}^{-1}\right) \text {, }
$$$$
=-n r^{n-1}\left(Q_{1}-(2 n+1) Q_{2}-Q_{3}-(2 n+1) Q_{4}\right)
$$

where $\zeta=(r, \theta), \xi=\cos \theta$. 